\title{
The evolution of thymic lymphomas in p53 knockout mice
}

\author{
Crissy Dudgeon, ${ }^{1,6}$ Chang Chan, ${ }^{1,2,6}$ Wenfeng Kang, ${ }^{1}$ Yvonne Sun, ${ }^{1}$ Ryan Emerson, ${ }^{3}$ Harlan Robins,,${ }^{3,4}$ \\ and Arnold J. Levine ${ }^{1,5}$

\begin{abstract}
${ }^{1}$ Rutgers Cancer Institute of New Jersey, New Brunswick, New Jersey 08901, USA; ${ }^{2}$ Department of Medicine, Rutgers Robert Wood Johnson Medical School, New Brunswick, New Jersey 08901, USA; ${ }^{3}$ Biomarker Discovery, Adaptive Biotechnologies, Seattle, Washington 98102, USA; ${ }^{4}$ Public Health Sciences, Fred Hutchinson Cancer Research Center, Seattle, Washington 98109, USA: ${ }^{5}$ Simons Center for Systems Biology, School of Natural Sciences, Institute for Advanced Study, Princeton, New Jersey 08540, USA
\end{abstract}

Germline deletion of the p53 gene in mice gives rise to spontaneous thymic (T-cell) lymphomas. In this study, the p53 knockout mouse was employed as a model to study the mutational evolution of tumorigenesis. The clonality of the T-cell repertoire from 533 knockout and wild-type thymic cells was analyzed at various ages employing TCRß sequencing. These data demonstrate that 53 knockout thymic lymphomas arose in an oligoclonal fashion, with tumors evolving dominant clones over time. Exon sequencing of tumor DNA revealed that all of the independently derived oligoclonal mouse tumors had a deletion in the Pten gene prior to the formation of the TCR $\beta$ rearrangement, produced early in development. This was followed in each independent clone of the thymic lymphoma by the amplification or overexpression of cyclin Ds and Cdk6. Alterations in the expression of Ikaros were common and blocked further development of CD-4/CD-8 $\mathrm{T}$ cells. While the frequency of point mutations in the genome of these lymphomas was one per megabase, there were a tremendous number of copy number variations producing the tumors' driver mutations. The initial inherited loss of p53 functions appeared to delineate an order of genetic alterations selected for during the evolution of these thymic lymphomas.

[Keywords: p53; T-cell lymphoma; mutation rate; clonality; Pten; chromosome abnormalities]

Supplemental material is available for this article.

Received September 3, 2014; revised version accepted October 27, 2014.

Mutations in the p53 gene arise in $>50 \%$ of all human cancers (Hollstein et al. 1991; Levine et al. 1991), and individuals with germline mutations in the TP53 gene develop Li-Fraumeni syndrome, a disorder that increases the risk of developing many different cancers, especially in children and young adults (Malkin 2011). The p53 protein functions as a transcription factor to induce cell cycle arrest, apoptosis, and senescence following genotoxic stress. It does this mainly by enhancing the transcription of an array of genes, such as $C d k n 1 a(\mathrm{p} 21)$ and $B B C 3$ (Puma), which carry out these cellular functions. Because of its abilities to protect cells from accumulating DNA damage following genotoxic stress, p53 has been described as the "guardian of the genome" (Lane 1992). Other cellular functions that p53 has been known to regulate transcriptionally include DNA repair, metabolism, autophagy, angiogenesis, and antioxidant potential (Levine and Oren 2009).

${ }^{6}$ These authors contributed equally to this work. Corresponding author: alevine@ias.edu

Article is online at http://www.genesdev.org/cgi/doi/10.1101/gad.252148.114 Freely available online through the Genes \& Development Open Access option.
Loss of p53 expression in p53 knockout mice reveals a role for $\mathrm{p} 53$ in the protection of mice from spontaneous tumorigenesis. The majority of p53 knockout mice succumb to thymic $\mathrm{CD}^{+} \mathrm{CD}^{+}$double-positive T-cell lymphomas (Donehower et al. 1995), but the events responsible for the formation and evolution of these tumors in p53 knockout mice remain poorly understood. For example, it is unclear what the mutation frequencies in these thymic lymphomas are during their development. Could these tumors arise from a single clone, or are they oligoclonal? If they are oligoclonal, do these clones compete, and will a dominant clone arise with time? What are the mutations that drive this process? Do all of the tumors that arise in different p53 knockout mice have the same driver genes altered to produce these tumors? Is the order in which these driver mutations arise important for the selection process in forming a lymphoma? All of these questions are addressed and answered in this study.

(c) 2014 Dudgeon et al. This article, published in Genes \& Development, is available under a Creative Commons License (Attribution 4.0 International), as described at http://creativecommons.org/licenses/by/4.0. 


\section{Results}

Assessing the clonality of thymic T cells in wild-type and p53 knockout T cells and thymic lymphomas

p53 knockout mice develop thymic lymphomas over the first $6 \mathrm{mo}$ of their lives. As each $\mathrm{T}$ cell arises in the thymus, it is marked with a unique T-cell receptor, and so one can assess the clonal lineages of a $\mathrm{T}$ cell by sequencing the V-D-J region of the DNA that defines a T cell and its clonal progeny (Robins et al. 2009; Gopalakrishnan et al. 2013). The number of identical V-D-J DNA sequencing reads is proportional to the number of offspring from each T-cell clone. To establish the baseline of unique copy and replicated T-cell clones in a thymus from wild-type C57Bl/6 mice, the DNA from a thymus was extracted from two male and two female mice at $17 \mathrm{~d}$ of embryonic life (E17) and $3 \mathrm{wk}, 6 \mathrm{wk}, 9 \mathrm{wk}$, and $20 \mathrm{wk}$ after birth. The PCR primers were located in the V region spanning to the constant region of the TCR- $\beta$ chain DNA. The PCR products were sequenced, and the number of unique T-cell clones was determined as well as the frequency of oligoclonal $\mathrm{T}$ cells in the thymus (Table 1 , the percentage is given for the two most common clones observed). Table 1 provides the frequency of T-cell clones in the thymus of a typical wild-type mouse. Possible PCR primer bias, which can alter the number of PCR copies that are then sequenced, was corrected by employing the algorithms provided by Adaptive Biotechnologies (Carlson et al. 2013). The highest frequency of replicated T-cell clones in the wild-type thymus varied between $0.08 \%$ and $0.17 \%$ of the total $\mathrm{T}$ cells sequenced (average of $0.11 \% \pm$ $0.05 \%$ ) (Table 1). In the thymus of the p53 knockout

Table 1. Clonality of wild-type (WT) and p53 knockout (p53-KO) thymus and thymic lymphomas at various ages

\begin{tabular}{|c|c|c|}
\hline & $\mathrm{WT}^{*}$ & p53-KO \\
\hline E17 & $\begin{array}{l}0.16 \%, 0.15 \% \\
0.17 \%, 0.17 \% \\
0.17 \%, 0.14 \% \\
0.16 \%, 0.15 \%\end{array}$ & $\begin{array}{l}0.16 \%, 0.14 \% \\
0.15 \%, 0.13 \% \\
0.25 \%, 0.20 \% \\
0.19 \%, 0.18 \%\end{array}$ \\
\hline 3 weeks & $\begin{array}{l}0.10 \%, 0.05 \% \\
0.07 \%, 0.07 \% \\
0.07 \%, 0.05 \% \\
0.06 \%, 0.05 \%\end{array}$ & $\begin{array}{l}0.07 \%, 0.07 \% \\
0.18 \%, 0.14 \%\end{array}$ \\
\hline 6 weeks & $\begin{array}{l}0.08 \%, 0.08 \% \\
0.16 \%, 0.13 \% \\
0.11 \%, 0.11 \%\end{array}$ & $\begin{array}{l}0.10 \%, 0.10 \% \\
0.11 \%, 0.11 \%\end{array}$ \\
\hline 9 weeks & $\begin{array}{l}0.08 \%, 0.07 \% \\
0.07 \%, 0.06 \% \\
0.16 \%, 0.14 \%\end{array}$ & $\begin{array}{c}2.9 \%, 1.2 \% \\
21 \%, 13.8 \%\end{array}$ \\
\hline 20 weeks & $\begin{array}{l}0.12 \%, 0.11 \% \\
0.15 \%, 0.09 \%\end{array}$ & $\begin{array}{l}70 \%, 28 \% \\
43 \%, 19 \% \\
70 \%, 5 \% \\
15 \%, 5 \%\end{array}$ \\
\hline
\end{tabular}

*The first number denotes the highest clone percentage, and the second number is the second-highest clone of the T-cell population in that particular thymus. mice, these numbers remained the same as wild-type mice from E17 through 6 wk after birth $10.14 \% \pm 0.054$; $P=0.54$, Student's $t$-test). By 9 wk after birth, the number of unique clones in the thymus began to decline, and the frequency of replicated clones increased (Fig. 1).

The increase in the frequency of replicated clones by 9 wk (30-fold to 200-fold) and 20 wk (120-fold to 600-fold) was a statistically significant difference from wild-type mice, and we employed this as the definition for determining that a thymic lymphoma had been formed and detected. The clonal expansion of these T cells is anomalous because they have not yet been exposed to antigen, so this could not be antigen-driven clonal expansion. Also, the loss of p53 itself did not cause a shift in the ratio of productive to nonproductive TCR $\beta$ sequences, as they remain similar in the 6-wk-old thymus (Supplemental Table 1). The ratio is reduced fourfold to fivefold in the p53 knockout thymic lymphomas, leading us to believe that another protein may be responsible for correct TCR $\beta$ selection.

The number of different clones (distinct TCR- $\beta$ chains) contributing to the thymic lymphoma in a single p53 knockout mouse demonstrated that these tumors arose multiple independent times and were oligoclonal. By 20 wk, dominant clones arose (Fig. 2) so that, in one case, $98 \%$ of the T cells in the thymus arose from two different clones. In other cases, as many as 10 different clones contributed to these tumors.

The clonal frequencies for the total population of E17, $3 \mathrm{wk}, 6 \mathrm{wk}, 9 \mathrm{wk}, 20 \mathrm{wk}$ wild-type and p53 knockout thymic $\mathrm{T}$ cells, including the p53 knockout thymic lymphomas at $20 \mathrm{wk}$, are available at http://sns.ias.edu/ $\sim$ cschan/TLymphoma.

\section{Whole-exome sequencing of p53 knockout thymic lymphomas}

Having established the timing of tumorigenesis in the p53 knockout thymic lymphoma model, we can now ascertain potential driving factors for these transformations. To explore this, whole-exome sequencing of three independent p53 knockout thymic lymphoma samples obtained at $20 \mathrm{wk}$ after birth was carried out. As a control sample of normal genomic DNA, matched tail DNA from a female C57Bl/6 mouse was employed. The nonsynonymous point mutations or stop codon/frameshift mutations detected by this sequencing did not identify any tumor driver mutations in similar genes between the three tumor DNA samples that were sequenced (Supplemental Table 2). Exon sequencing data are available at http://sns.ias.edu/ cschan/TLymphoma.

\section{Copy number variations (CNVs) in p53 knockout thymic lymphomas}

Using the exome sequencing data, CNVs occurring within each of the three tumors sequenced were determined. Using a cutoff of -0.3 for deletions (corresponding to $38 \%$ of the tumor sample having deletion of one copy) and 0.2 for amplification (corresponding to $30 \%$ of the tumor sample having amplification of one copy), there are 276, 348, and 422 CNVs in the three tumors 
A

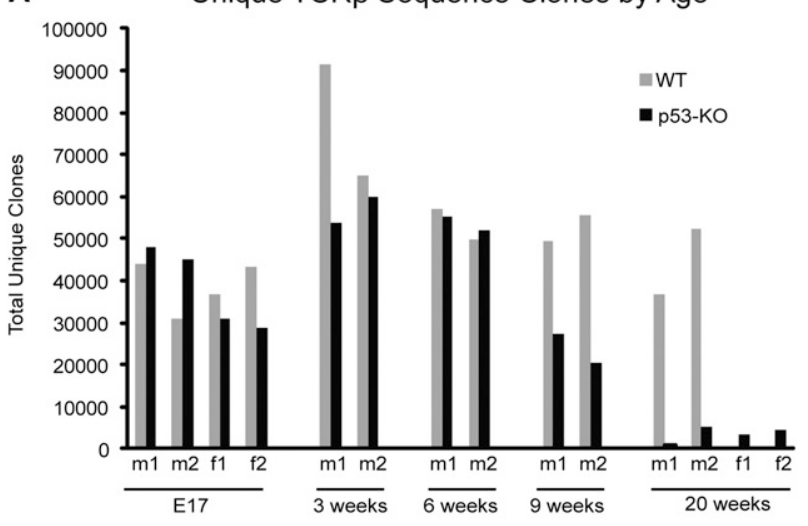

B

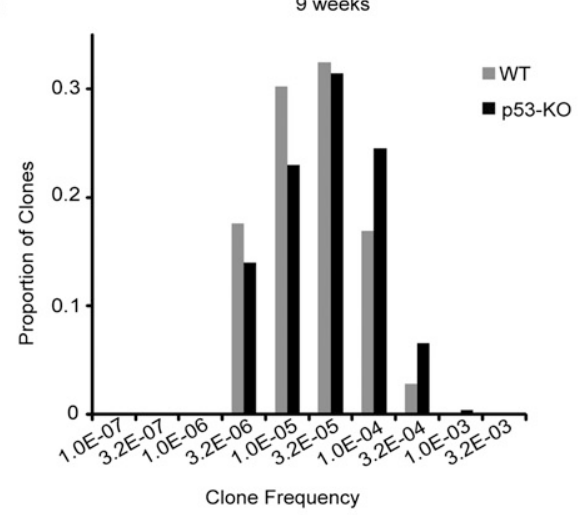

C

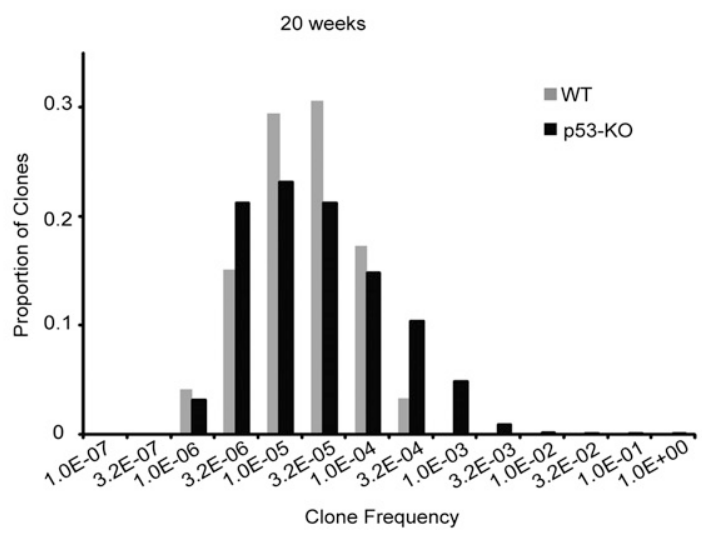

Figure 1. T-cell clonality decreases in p53 knockout (p53-KO) thymus. (A) The total number of unique clones represents the number of TCR $\beta$ sequencing reads that are different within a sample. $(\mathrm{m})$ Male; (f) female. $(B, C)$ Histograms showing distribution of clones binned by their clonal frequencies at $9 \mathrm{wk}$ and $20 \mathrm{wk}$.

evaluated (Fig. 3). In contrast to the point mutations, these CNVs provided reproducible patterns of losses or gains of DNA copies. Of these, identical biallelic Pten deletions were found in every oligoclonal tumor within an individual mouse. However, individual p53 knockout mice had a different Pten deletion found in each of their oligoclonal tumors. The tumor of a male mouse with thymic lymphomas at $20 \mathrm{wk}$ was composed of clones representing $39.4 \%, 13.5 \%, 8.4 \%$, and $7.0 \%$ of the total tumor load, but the identical Pten deletion was represented by $85 \%$ of the DNA reads. In a second male mouse, the tumor was composed of two major clones at $76.7 \%$ and $9.7 \%$, and $98 \%$ of the DNA reads had the same Pten deletion. These data are consistent with the idea that in each p53 knockout mouse, the Pten deletion occurred and was selected for future tumors during development prior to the creation of the TCR $\beta$ rearrangement and expression of the protein on the T-cell surface. In 10 tumors analyzed for Pten protein by Western blot, Pten deletion was found in seven, with three tumors having reduced levels of Pten (Fig. 4A). Other recurrent deletions were observed at lower frequencies in tumors and are listed in Supplemental Table 3. Amplifications and chromosomal aneuploidy were more difficult to interpret because there was no single gene or gene cluster with a significantly high copy number. Many of the amplification regions encompassed the entire chromosome (Fig. 3), which include chromosomes 2, 5, 9, 11, 12, 14, 15, and 16. Of these, only chromosome 5 was amplified in all three of the tumor samples. Taken together, there were 175 genes that are recurrently amplified in all tumors (Supplemental Table 4). These amplifications occurred in a smaller percentage of clones, unlike the Pten deletion, suggesting that these driver amplifications followed after TCR $\beta$ rearrangement. A comparison of this list with known human cancer driver genes revealed Cdk6 and Ikzf1 (Ikaros) as two genes whose amplifications may drive tumorigenesis.

\section{Pten, Cyclin D, Cdk6, and Ikaros expression in p53} knockout thymic lymphomas

Western blotting confirmed the loss or reduction of Pten expression in all p53 knockout thymic lymphomas samples when compared with normal thymus tissue or thymic protein from p53 knockout mice at $6 \mathrm{wk}$ after birth, when lymphoma cells are not yet detectable and normal cells far exceed malignant cells (Fig. 4A). The Western blot employing antibodies to Cdk6 demonstrates that this gene is amplified and overexpressed in all 10 tumor samples compared with normal thymic $\mathrm{T}$ cells (Fig. 4B). Since Cdk6 requires the binding of Cyclin D to be fully functional, the levels of Cyclin D1, Cyclin D2, and Cyclin D3 in the tumors were determined. A very high expression of at least one type of Cyclin D for each tumor along with inactivation or loss of $\mathrm{Rb}$ in three tumors were observed (Fig. 4C). The Western blot employing antibodies against Ikaros, a transcription factor required for differentiation of thymic T cells, demonstrated dominant-negative isoforms of Ikaros (IK4-8) in seven of the tumors. Ikaros was found to be deleted in one tumor (Fig. 4D). The levels of the Hes 1 protein (Kathrein et al. 2008; Kleinmann et al. 2008) were overexpressed in seven of the tumors, suggesting a pseudoactivation of the Notch1 pathway due to overexpression of Ik4-8 (Fig. 4D). Analysis of these same proteins in thymic lymphomas from the 53 mutant (R172H/R172H) revealed a similar expression profile (Supplemental Fig. 1). Taken together, these data support a role for the loss of p53 (genomic instability), the loss of Pten (altered metabolic regulation), the overexpres- 
A p53-KO tumor female 1

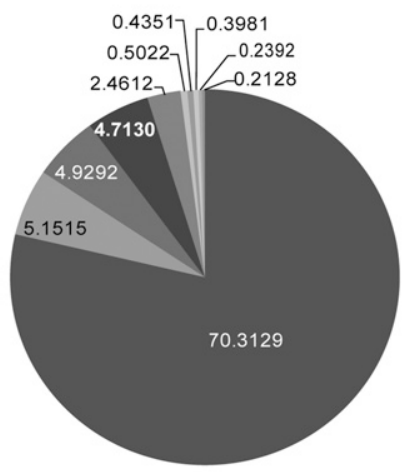

C

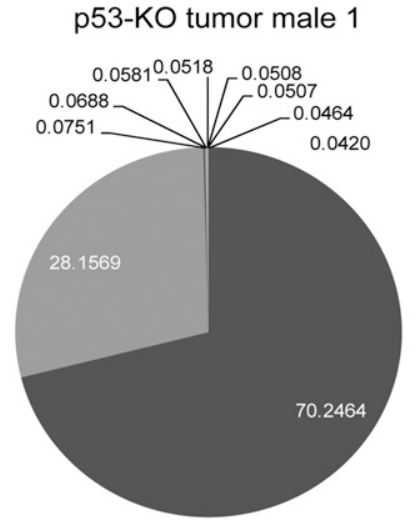

B p53-KO tumor female 2

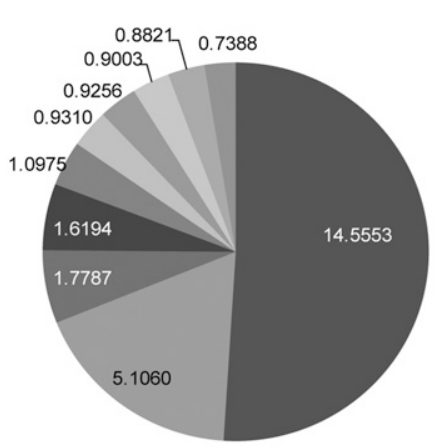

D

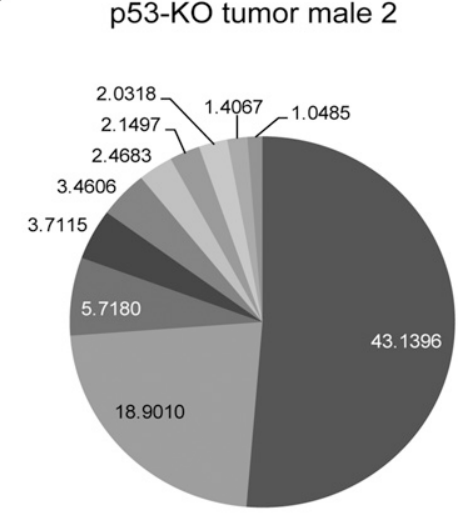

Figure 2. Oligoclonal p53 knockout (p53-KO) thymic lymphomas are comprised of a few dominant clones. $(A-D)$ The top 10 clones for each thymic lymphoma were plotted in a pie chart to show dominant clones. The percentages for all clones can be seen at http://sns. ias.edu/ cschan/TLymphoma. sion of Cyclin D-Cdk6 (cell cycle regulation), and altered splicing patterns of Ikaros (altering T-cell developmental pathways) in driving cellular proliferation and altering development during mouse T-cell lymphomagenesis.

\section{Discussion}

Since all exonic mutations were sequenced in the tumors, we were able to estimate a mutation frequency for the p53 knockout thymic lymphoma. An average for the three tumors sequenced produced $\sim 100$ point mutations found per $100 \mathrm{Mb}$ of genomic DNA, giving a somatic point mutation frequency of one mutation per megabase of DNA. This somatic mutation frequency is similar to the mutational frequency observed with human breast cancers and pancreatic cancers (Lawrence et al. 2013). On the other hand, the frequency of CNVs detected in these tumors is very high, between 276 and 422 per genome for the three tumors. Two of the tumors have CNV patterns resembling chromothripsis, a pattern observed in human tumors with p53 mutations. The number of distinct clones of $\mathrm{T}$ cells contributing to a thymic lymphoma in a mouse between 9 wk and 20 wk after birth was $\sim 0.13-$ 0.8 clones produced per day, reflecting the high frequency of CNVs observed. It is very likely that these numbers represent minimal estimates per day due to large variations caused naturally between mice and over time because of selective pressures for the most robust clones. While some mutations were common to most of these clones (Pten deletion, Cyclin D-Cdk6 overexpression, and Ikaros splicing changes), other mutations arising later in tumor evolution (by 20 wk after birth) produced dominant clones of $\mathrm{T}$ cells contributing to these lymphomas at later times. The high frequency of genomic instability (CNVs) driven by the inherited p53 mutations demonstrates the importance of p53 to the genetic integrity of somatic T cells.

Starting with a germline deletion of the p53 gene, the great majority of thymic lymphomas that are produced are a result of mutations in the same genes, which appear to be selected for in the same order of temporal events. Following the inherited p53 mutation, a Pten mutation is rapidly selected for prior to the development of mature $\mathrm{T}$ cells with receptors, which may also explain the decrease in the ratio of productive to nonproductive TCR $\beta$ sequences (Newton and Turka 2012). While the high mutation frequency in the absence of p53 gives rise to many types of mutations, the loss of Pten metabolically permits the selection of those cells during development in the T-cell lineage (and possibly other lineages) because of its role in the insulin-like growth factor pathway. This selective advantage would increase activated Akt, glucose uptake, and metabolic precursors needed for cell division. Then, after the T-cell receptor is added to the surface of $\mathrm{T}$ cells, additional mutations arise, such as the dominant-negative isoforms of IK4-8, which prevents terminal differentiation in this lineage. This might well then be followed by the overexpression of 
A

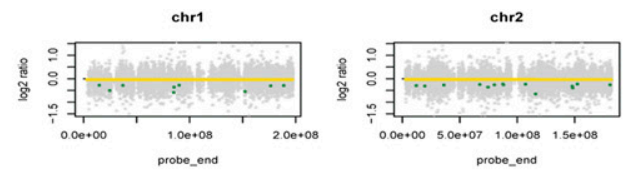

chr6

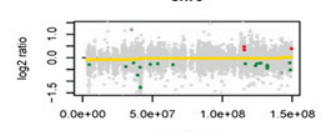

probe_end

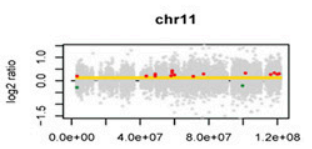

probe_end

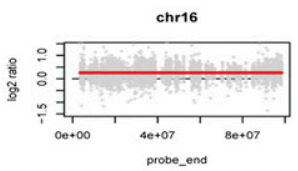

B
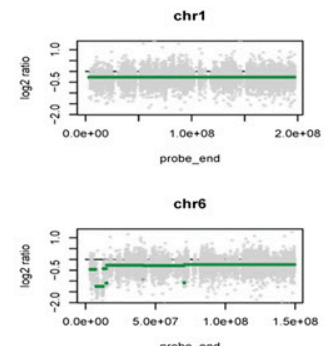

chr11

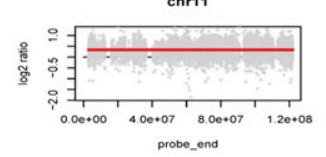

chr16
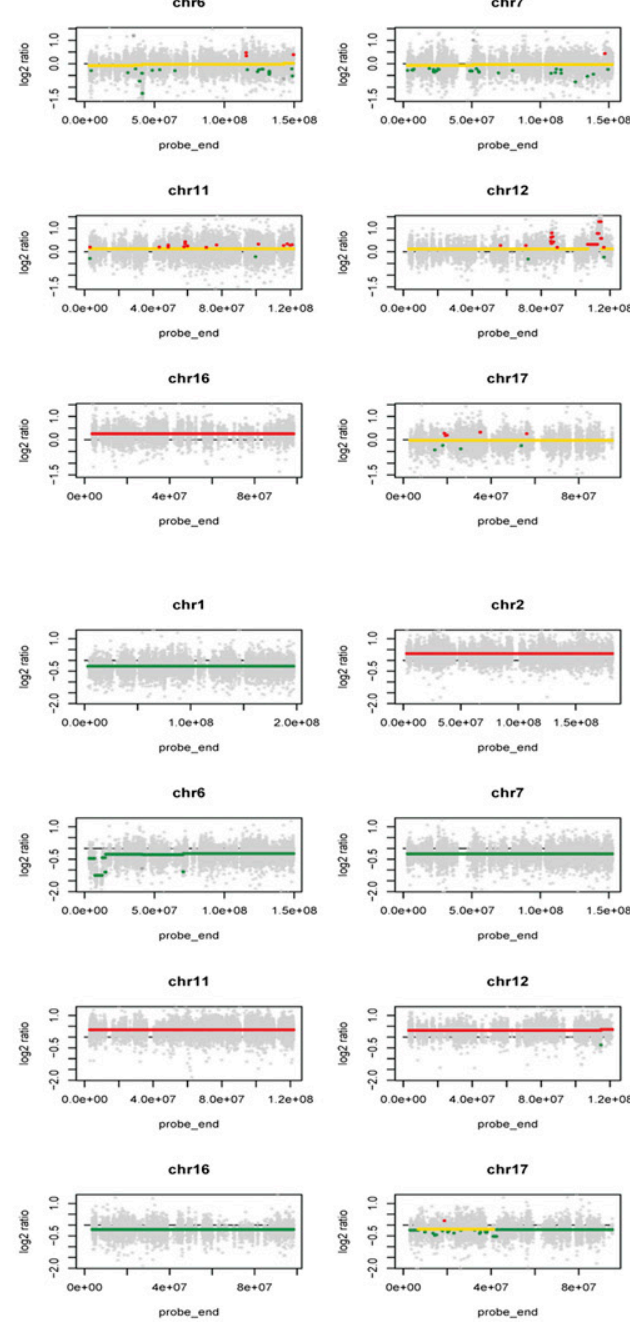

probe_end

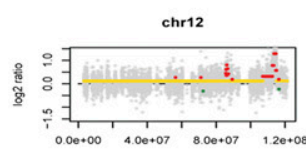

probe_end
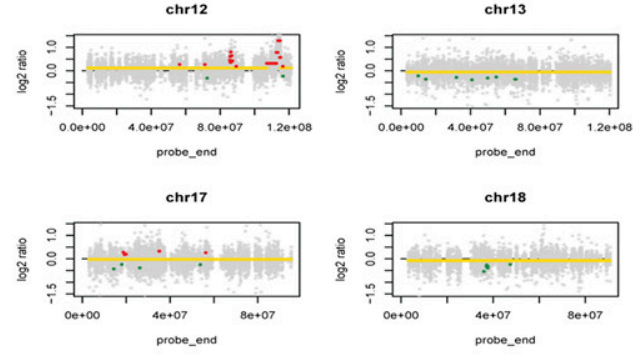

probe_end

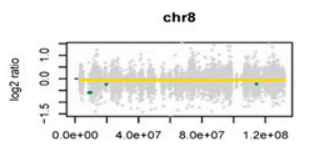

probe_end
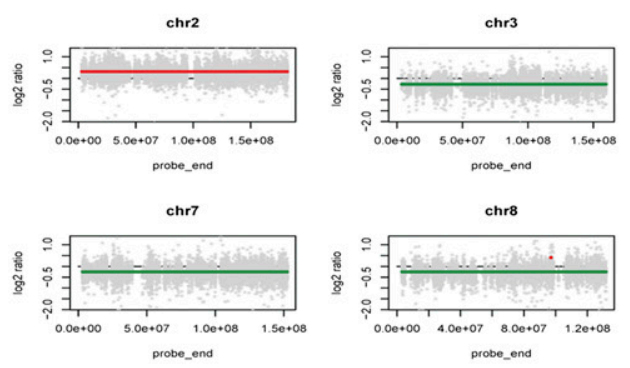

chr12
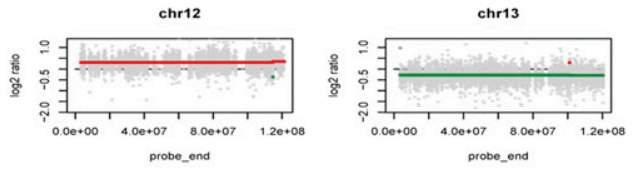

chr17
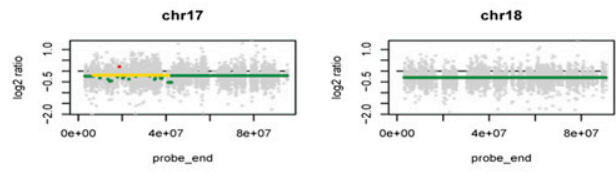

probe_end

C
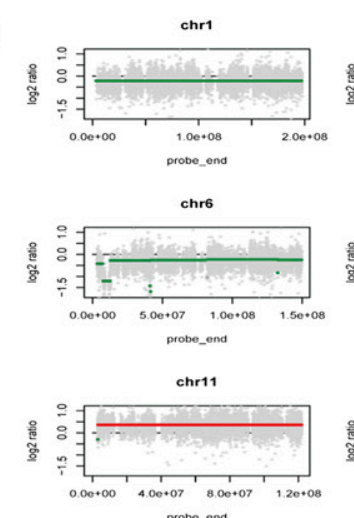

probe_end
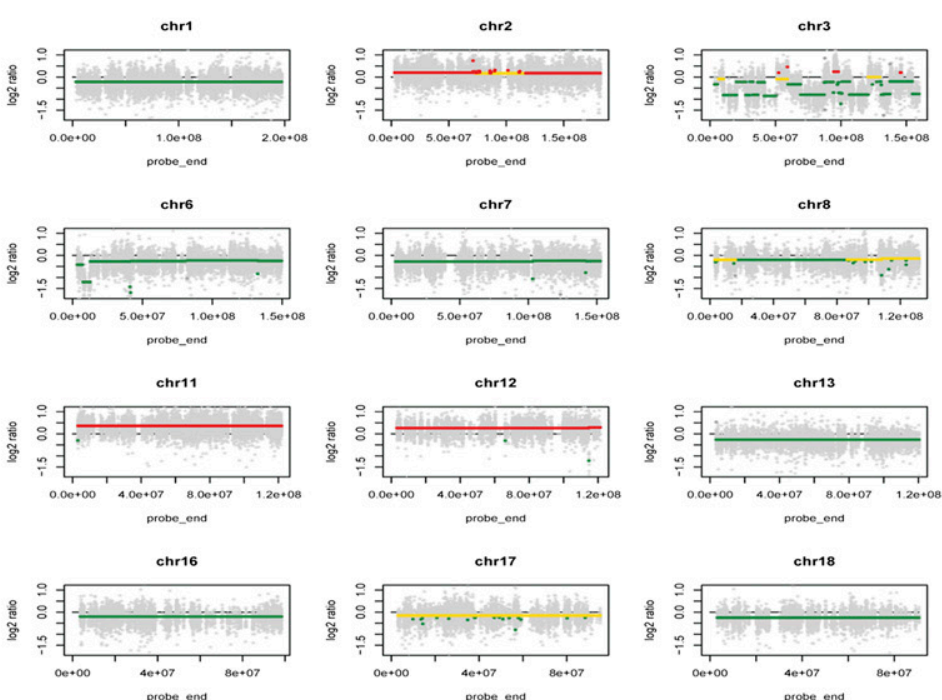

chr7
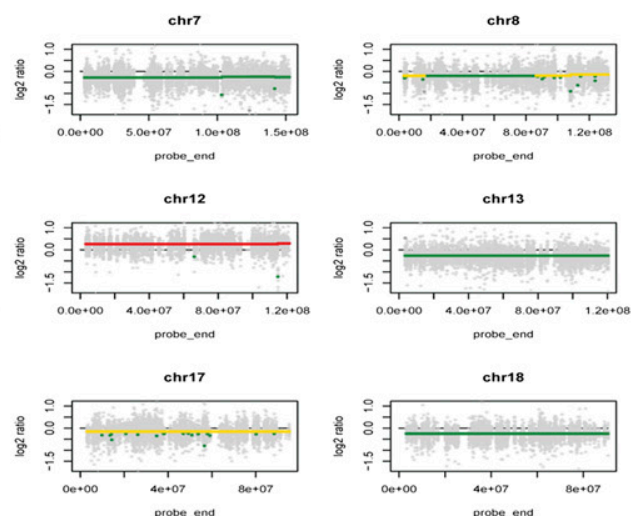

chr18

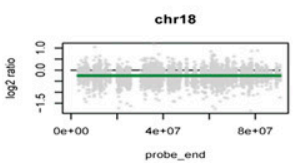

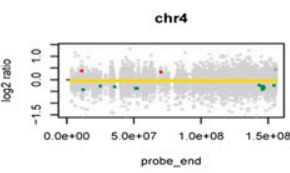
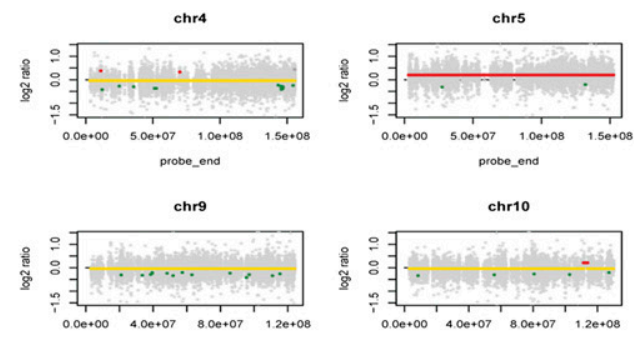

probe_end
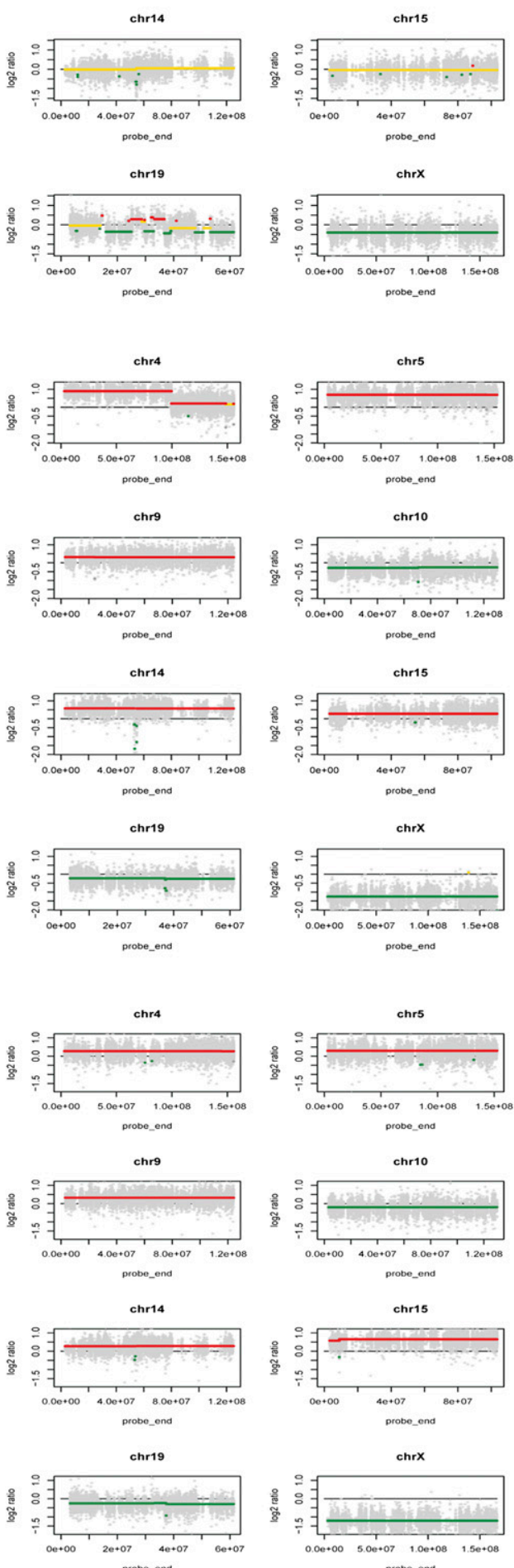

Figure 3. Increased CNVs in p53 knockout (p53-KO) thymic lymphomas. CNV analysis was completed as described in the Materials and Methods. CNV plots for female 1 p53 knockout thymic lymphoma $(A)$, male 1 p53 knockout thymic lymphoma $(B)$, and male 2 p53 knockout thymic lymphoma $(C)$ are shown. Red lines indicate areas of amplification, yellow lines indicate no change, and green lines are areas of deletion. 
A

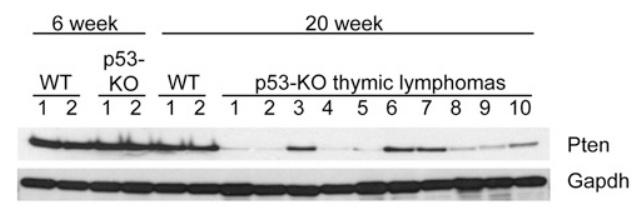

B

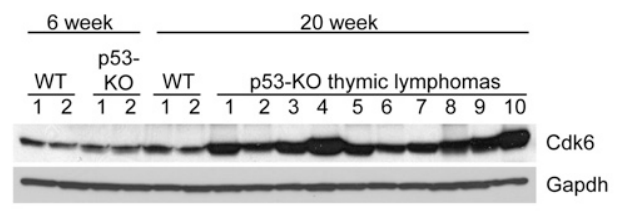

C

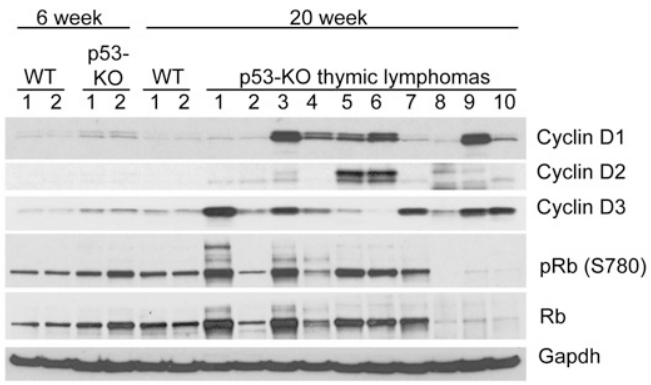

D

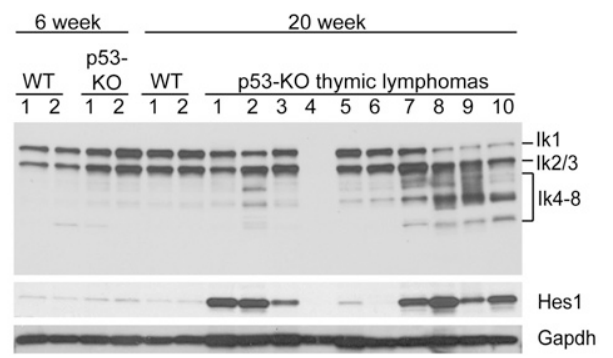

Figure 4. Pten loss, Cdk6 overexpression, and dominant-negative expression in p53 knockout (p53-KO) thymic lymphomas. Western blot of 6-wk-old wild-type (WT) and p53 knockout thymus and 20-wk-old wild-type and p53 knockout thymic lymphoma protein indicating the expression of Pten $(A)_{;}$Cdk6 $(B)$; Cyclin D1, Cyclin D2, Cyclin D3, phospho-Rb, and Rb $(C)$; and Ikaros isoforms (Ik1-8) and Hes1 (D), with Gapdh as a loading control $(A-D)$.

Cyclin D1, Cyclin D2, and Cyclin D3, which are clearly observed after the T-cell receptor is present, as is the Cdk6 amplification or $\mathrm{Rb}$ deletion. While Pten deletion and Cdk6 overexpression are found in all tumors, the choice of which D cyclin is overproduced and whether Ikaros dominant-negative isoforms are made occurs in some but not all tumors. These observations suggest that the presence of a germline p53 mutation sets an order to the selection of possible genes that contribute to the tumor not only when dominant clones arise but, in some cases, like Pten, for all clones that contribute to the tumor.

The genes that we identified are well known to drive T-cell leukemogenesis in mice as well as in humans. PTEN, CDK6, CCNDs, RB, and dominant-negative Ikaros isoform expression has been previously shown to be altered in human T-cell leukemias/lymphomas /Chilosi et al. 1998; Sun et al. 1999; Hatta and Koeffler 2002;
Sicinska et al. 2003; Maser et al. 2007; Li et al. 2008; Gutierrez et al. 2009). Although we did not identify activating Notch 1 mutations in the p53 knockout lymphoma that are common in T-ALL (T-cell adult lymphoblastic leukemia) (Weng et al. 2004; O'Neil et al. 2006), aberrant expression of Ikaros dominant-negative isoforms seems to substitute. Also, while p53 is normally wild type in T-ALL, Arf is biallelically deleted (Ferrando et al. 2002; Mullighan et al. 2008). However, by starting with a loss of p53 in our model, it appears that we removed the selective pressure for Arf deletion. Therefore, in this case, a p53 mutation functions as a gatekeeper mutation that sets a path of subsequent mutations and selection events leading rapidly and with a high frequency to oligoclonal tumors, providing the tumor heterogeneity that finally selects dominant clones. We will need to understand both the path chosen and the nature of the subsequent mutations that produce dominant clones. This model system provides that opportunity.

\section{Materials and methods}

\section{Mice}

All animal protocols used in this study were approved by the Rutgers Biomedical and Health Sciences Animal Care and Use Committee. C57BL/6 wild-type and p53 knockout mice were housed in a room maintained at $75^{\circ} \mathrm{F}$ and $50 \%$ humidity with 12-h light/dark cycles in microisolator cages and allowed access to water and chow ad libitum. For extraction of thymi, wild-type and p53 knockout mice were euthanized at the indicated times using $\mathrm{CO}_{2}$, and tissue was placed in liquid nitrogen for further analysis.

\section{DNA extraction and TCR $\beta$ sequencing}

DNA was extracted from $30 \mathrm{mg}$ of thymic tissue using the Purelink genomic DNA extraction (Life Technologies). The TCR $\beta$ locus was sequenced using the ImmunoSeq survey level assay by Adaptive Biotechologies with $500 \mathrm{ng}$ of genomic DNA from wild-type and p53 knockout mice at various ages, including p53 knockout thymic lymphomas. TCR $\beta$ sequencing was analyzed using the ImmunoSeq Analyzer (Adaptive Biotechnologies).

\section{Exome sequencing and somatic point mutation analysis}

Whole-exome sequencing and SNP/indel calling were completed by BGI Americas, Inc., using $3 \mu \mathrm{g}$ of genomic DNA from p53 knockout thymic lymphomas female 1 , male 1 , and male 2 . The tail DNA from female 1 , taken at 3 wk of age, was used as a matched normal for all tumors. The exonic regions were captured using Agilent SureSelect Mouse All Exon $50 \mathrm{Mb}$ and sequenced by 100-base-pair (bp) paired-end reads on an Illumina platform. The $\mathrm{mm} 9$ mouse reference genome was used to align good quality reads using Burrows-Wheeler alignment (BWA). The genome analysis toolkit (GATK) best practices workflow was followed to preprocess data. Sorting, merging, and indexing of the sequencing reads were accomplished with SAMtools 0.1.16. Picard 1.100 was used to mark PCR duplicates. To correct the misalignment caused by indels, local realignment was accomplished using RealignerTargetCreator and IndelRealigner of GATK (McKenna et al. 2010). The exome interval list (provided by the Agilent SureSelect Mouse All Exon kit) was used to identify the 
position of each exon in realignment. Recalibration of the base quality score was completed using GATK BaseRecalibrator. Somatic point mutations were called on matched normal and tumor BAM files using MuTect (Cibulskis et al. 2013). After identifying somatic genomic variants, known SNPs were identified by comparing with dbSNP128 for mice. After filtering known SNPs, somatic mutations were found in tumor samples as compared with normal samples.

\section{Structural variation analysis}

CNV analysis was accomplished using ExomeCNV (Sathirapongsasuti et al. 2011) using the default parameter setting with the sample admixture rate set to 0.5. Briefly, DepthOfCoverage in GATK was used to convert BAM files (after removing PCR duplicates) into coverage files. The logarithm of coverage ratio between tumor and normal samples was calculated by ExomeCNV. ExomeCNV used this log ratio of read depth at each exon to call exonic CNVs and combine them into segments using the circular binary segmentation (CBS) algorithm (Olshen et al. 2004). Chromosome Y was not specified in our CNV analysis because we used the same female normal sample matched for both female and male tumor samples.

\section{Western blotting}

Ten milligrams of tissue from thymus or thymic lymphomas was homogenized in $500 \mu \mathrm{L}$ of $2 \times$ Laemmli buffer $(0.125 \mathrm{M}$ Tris-HCl at $\mathrm{pH} 6.8,10 \% \beta$-mercaptoethanol, $4 \%$ sodium dodecyl sulphate, $20 \%$ glycerol, $0.05 \%$ bromophenol blue). Ten microliters of protein extract was subjected to SDS-PAGE and transferred to an Immobilon-P membrane (EMD Millipore). Western blot was carried out using antibodies to Pten (\#9188), Cdk6 (\#3136), Cyclin D1 (\#2978), Cyclin D2 (\#3741), Cyclin D3 (\#2936), phospho-Rb (S780) (\#9307), Rb (\#9313), Ikaros (\#5443), and Hes1 (\#11988) (Cell Signaling) and Gapdh (\#25778) (Santa Cruz Biotechnology, Inc.).

\section{Competing interest statement}

The authors disclose no potential conflicts of interest.

\section{Acknowledgments}

This study was funded by a National Institutes of Health PO1 grant (CA087497 to A.J.L.).

\section{References}

Carlson CS, Emerson RO, Sherwood AM, Desmarais C, Chung MW, Parsons JM, Steen MS, LaMadrid-Herrmannsfeldt MA, Williamson DW, Livingston RJ, et al. 2013. Using synthetic templates to design an unbiased multiplex PCR assay. Nat Commun 4: 2680.

Chilosi M, Doglioni C, Yan Z, Lestani M, Menestrina F, Sorio C, Benedetti A, Vinante F, Pizzolo G, Inghirami G. 1998. Differential expression of cyclin-dependent kinase 6 in cortical thymocytes and T-cell lymphoblastic lymphoma/ leukemia. Am J Pathol 152: 209-217.

Cibulskis K, Lawrence MS, Carter SL, Sivachenko A, Jaffe D, Sougnez C, Gabriel S, Meyerson M, Lander ES, Getz G. 2013. Sensitive detection of somatic point mutations in impure and heterogeneous cancer samples. Nat Biotechnol 31: 213219.

Donehower LA, Harvey M, Vogel H, McArthur MJ, Montgomery CA Jr, Park SH, Thompson T, Ford RJ, Bradley A. 1995. Effects of genetic background on tumorigenesis in p53-deficient mice. Mol Carcinog 14: 16-22.

Ferrando AA, Neuberg DS, Staunton J, Loh ML, Huard C, Raimondi SC, Behm FG, Pui CH, Downing JR, Gilliland DG, et al. 2002. Gene expression signatures define novel oncogenic pathways in $\mathrm{T}$ cell acute lymphoblastic leukemia. Cancer Cell 1: 75-87.

Gopalakrishnan S, Majumder K, Predeus A, Huang Y, Koues OI, Verma-Gaur J, Loguercio S, Su AI, Feeney AJ, Artyomov MN, et al. 2013. Unifying model for molecular determinants of the preselection V $\beta$ repertoire. Proc Natl Acad Sci 110: E3206-E3215.

Gutierrez A, Sanda T, Grebliunaite R, Carracedo A, Salmena L, Ahn Y, Dahlberg S, Neuberg D, Moreau LA, Winter SS, et al. 2009. High frequency of PTEN, PI3K, and AKT abnormalities in T-cell acute lymphoblastic leukemia. Blood 114: 647-650.

Hatta Y, Koeffler HP. 2002. Role of tumor suppressor genes in the development of adult T cell leukemia/lymphoma (ATLL). Leukemia 16: 1069-1085.

Hollstein M, Sidransky D, Vogelstein B, Harris CC. 1991. p53 mutations in human cancers. Science 253: 49-53.

Kathrein KL, Chari S, Winandy S. 2008. Ikaros directly represses the notch target gene Hes1 in a leukemia $\mathrm{T}$ cell line: implications for CD4 regulation. I Biol Chem 283: 1047610484.

Kleinmann E, Geimer Le Lay AS, Sellars M, Kastner P, Chan S. 2008. Ikaros represses the transcriptional response to Notch signaling in T-cell development. Mol Cell Biol 28: 7465-7475.

Lane DP. 1992. Cancer. p53, guardian of the genome. Nature 358: $15-16$.

Lawrence MS, Stojanov P, Polak P, Kryukov GV, Cibulskis K, Sivachenko A, Carter SL, Stewart C, Mermel CH, Roberts SA, et al. 2013. Mutational heterogeneity in cancer and the search for new cancer-associated genes. Nature 499: 214218.

Levine AJ, Oren M. 2009. The first 30 years of p53: growing ever more complex. Nat Rev Cancer 9: 749-758.

Levine AJ, Momand J, Finlay CA. 1991. The p53 tumour suppressor gene. Nature 351: 453-456.

Li X, Gounari F, Protopopov A, Khazaie K, von Boehmer H. 2008. Oncogenesis of T-ALL and nonmalignant consequences of overexpressing intracellular NOTCH1. I Exp Med 205: 2851-2861.

Malkin D. 2011. Li-Fraumeni syndrome. Genes Cancer 2: 475 484.

Maser RS, Choudhury B, Campbell PJ, Feng B, Wong KK, Protopopov A, O'Neil J, Gutierrez A, Ivanova E, Perna I, et al. 2007. Chromosomally unstable mouse tumours have genomic alterations similar to diverse human cancers. $\mathrm{Na}$ ture 447: 966-971.

McKenna A, Hanna M, Banks E, Sivachenko A, Cibulskis K, Kernytsky A, Garimella K, Altshuler D, Gabriel S, Daly M, et al. 2010. The genome analysis toolkit: a MapReduce framework for analyzing next-generation DNA sequencing data. Genome Res 20: 1297-1303.

Mullighan CG, Miller CB, Radtke I, Phillips LA, Dalton J, Ma J, White D, Hughes TP, Le Beau MM, Pui CH, et al. 2008. BCRABL1 lymphoblastic leukaemia is characterized by the deletion of Ikaros. Nature 453: 110-114.

Newton RH, Turka LA. 2012. Regulation of T cell homeostasis and responses by pten. Front Immunol 3: 151.

Olshen AB, Venkatraman ES, Lucito R, Wigler M. 2004. Circular binary segmentation for the analysis of array-based DNA copy number data. Biostatistics 5: 557-572.

O'Neil J, Calvo J, McKenna K, Krishnamoorthy V, Aster JC, Bassing CH, Alt FW, Kelliher M, Look AT. 2006. Activating 
Dudgeon et al.

Notch1 mutations in mouse models of T-ALL. Blood 107: 781-785.

Robins HS, Campregher PV, Srivastava SK, Wacher A, Turtle CJ, Kahsai O, Riddell SR, Warren EH, Carlson CS. 2009. Comprehensive assessment of T-cell receptor $\beta$-chain diversity in $\alpha \beta$ T cells. Blood 114: 4099-4107.

Sathirapongsasuti JF, Lee H, Horst BA, Brunner G, Cochran AJ, Binder S, Quackenbush J, Nelson SF. 2011. Exome sequencing-based copy-number variation and loss of heterozygosity detection: ExomeCNV. Bioinformatics 27: 2648-2654.

Sicinska E, Aifantis I, Le Cam L, Swat W, Borowski C, Yu Q, Ferrando AA, Levin SD, Geng Y, von Boehmer H, et al. 2003. Requirement for cyclin D3 in lymphocyte development and T cell leukemias. Cancer Cell 4: 451-461.

Sun L, Crotty ML, Sensel M, Sather H, Navara C, Nachman J, Steinherz PG, Gaynon PS, Seibel N, Mao C, et al. 1999. Expression of dominant-negative Ikaros isoforms in T-cell acute lymphoblastic leukemia. Clin Cancer Res 5: 21122120.

Weng AP, Ferrando AA, Lee W, Morris JP, Silverman LB, Sanchez-Irizarry C, Blacklow SC, Look AT, Aster JC. 2004. Activating mutations of NOTCH1 in human T cell acute lymphoblastic leukemia. Science 306: 269-271. 


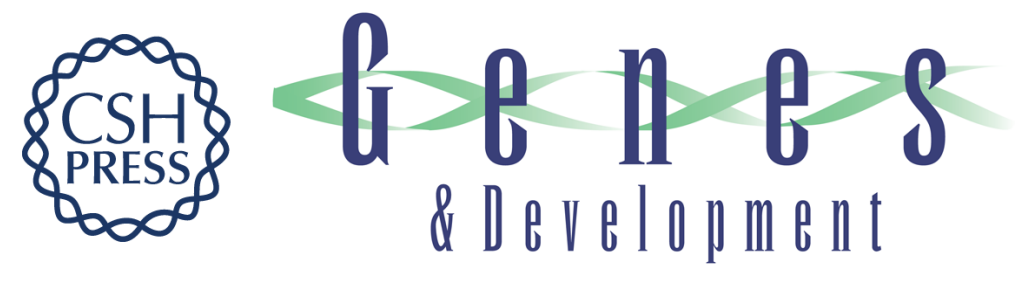

\section{The evolution of thymic lymphomas in p53 knockout mice}

Crissy Dudgeon, Chang Chan, Wenfeng Kang, et al.

Genes Dev. 2014, 28:

Access the most recent version at doi:10.1101/gad.252148.114

\section{Supplemental http://genesdev.cshlp.org/content/suppl/2014/12/01/28.23.2613.DC1 \\ Material \\ References This article cites 28 articles, 11 of which can be accessed free at: http://genesdev.cshlp.org/content/28/23/2613.full.html\#ref-list-1 \\ Creative This article, published in Genes \& Development, is available under a Creative Commons Commons License (Attribution 4.0 International), as described at License http://creativecommons.org/licenses/by/4.0. \\ Email Alerting Receive free email alerts when new articles cite this article - sign up in the box at the top Service right corner of the article or click here.}

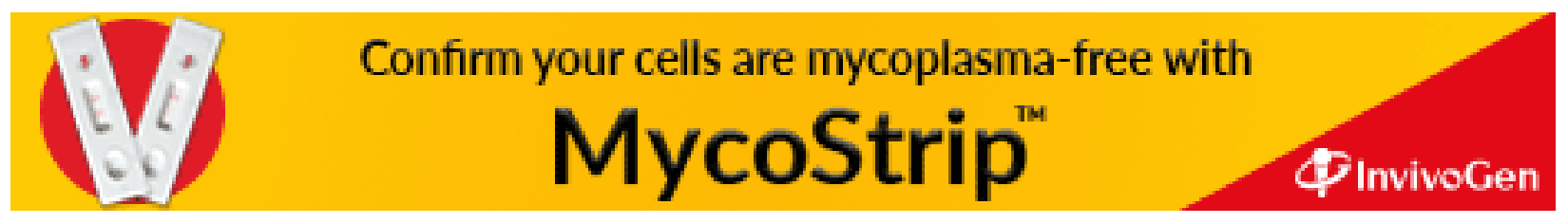

